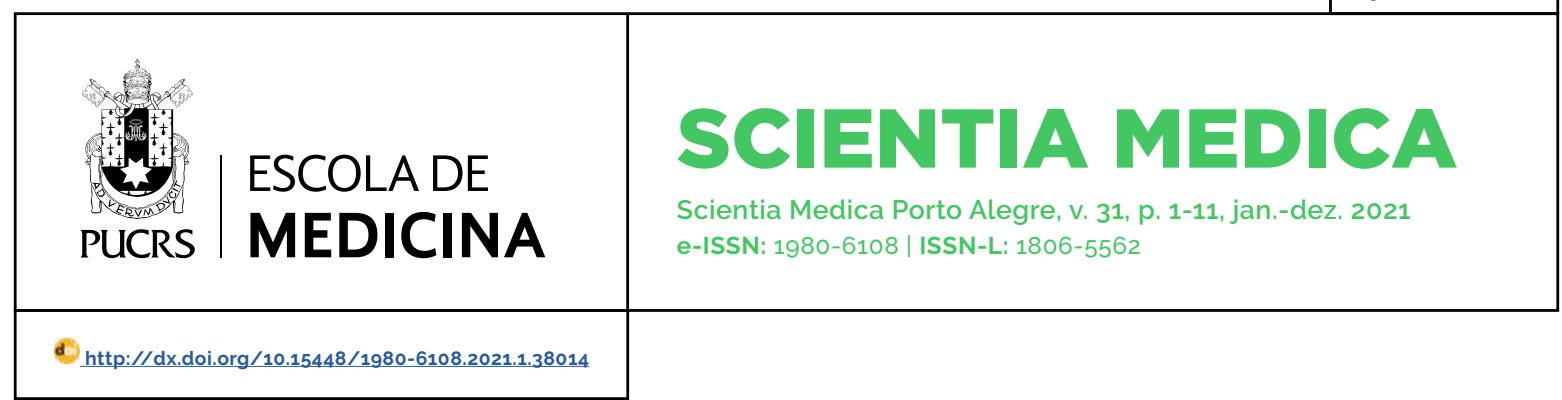

ARTIGO ORIGINAL

\title{
Fatores associados à realização de mamografia de acordo com dois critérios
}

\author{
Factors associated to mammography exam according to two criteria
}

\section{Kevin Francisco Durigon Meneghini ${ }^{1}$ \\ 0000-0002-2231-7389 \\ kevinmeneghini@hu.furg.br}

\section{Arnildo Agostinho \\ Hackenhaar ${ }^{2}$ \\ 0000-0003-1428-1328 \\ arnildo@vetorial.net}

\section{Samuel Carvalho Dumith ${ }^{3}$}

0000-0002-5994-735X

scdumith@yahoo.com.br

Recebido em: 9/5/2020

Aprovado em: 15/12/2020.

Publicado em: 04/02/2021.

\begin{abstract}
Resumo
Objetivo: objetivou-se comparar as prevalências e os fatores associados à realização de mamografia de acordo com dois critérios em vigência no Brasil.

Métodos: estudo transversal, de base populacional, com mulheres entre $40 \mathrm{e}$ 74 anos de Rio Grande, RS, Brasil. A cobertura de mamografia foi avaliada: 1) rastreamento anual para mulheres entre 40 e 74 anos; 2) rastreamento bienal para mulheres entre 50 e 69 anos. Os dados foram analisados por regressão de Poisson.

Resultados: participaram do estudo 413 (critério 1) e 246 (critério 2) mulheres. A cobertura de mamografia pelo critério 1 foi de 49,4\% (IC95\% 43,8 a 55,0), oscilando de um quarto para as que não consultaram um médico no último ano até dois terços para aquelas com maior escolaridade. Segundo o critério 2, a cobertura foi de $65.5 \%$ (IC95\% 59,2 a 71,7), indo de um terço entre as que não consultaram um médico no último ano a três quartos entre as obesas.
\end{abstract}

Conclusões: a cobertura de mamografia diferiu conforme o critério empregado. Maior nível socioeconômico e ter consultado com médico no último ano foram os fatores mais associados, independentemente do critério.

Palavras-chave: saúde pública, mamografia, programas de rastreamento, saúde da mulher, neoplasias da mama, prevenção secundária.

\section{Abstract}

Aims: we aimed to compare the prevalence and factors associated to mammography exam according to two Brazilian criteria.

Methods: a cross-sectional, population-based study, with women between 40 and 74 years old from Rio Grande, RS, Brazil. The coverage of mammography was evaluated: 1) annual screening for women aged 40 to 74 years old; 2) biennial screening for women aged 50 to 69 years old. Data analyses were performed by Poisson regression.

Results: it was included 413 (criterion 1) and 246 (criterion 2) women. The mammography coverage by criterion 1 was $49.4 \%(95 \% \mathrm{Cl} 43.8$ to 55.0$)$, ranging from a quarter for those who did not visit a doctor in the last year to two thirds for those with higher education level. Considering the criterion 2, the coverage was 65.5\% (Cl95\% 59.2 to 71.7), ranging from one-third among those who did not visit a doctor in the last year to three-quarters among obese women.

Conclusions: the mammography coverage differed according to the criterion considered. Higher socioeconomic status and having visiting a doctor in the last year were the most associated factors, regardless of the criterion.

Keywords: public health, mammography, mass screening, women's health, breast neoplasms, secondary prevention. 


\section{Introdução}

O câncer de mama impacta 2,1 milhões de mulheres anualmente, sendo a maior causa mundial de morte câncer-relacionada nessa população. A Organização Mundial da Saúde estimou 627 mil mortes em 2018, representando, $15 \%$ do total de mortes entre as mulheres (1). No Brasil, o Instituto Nacional de Câncer ponderou para 2018, aproximadamente, 60 mil novos casos, com taxas de incidência entre 45,02 e 68,78/100.000 e de mortalidade entre 10,31 e 17,55/100.000 nas regiões Sul e Sudeste (2).

Tendo como principais tipos os carcinomas ductal e lobular, encontrados em 80\% e 10\% dos casos, respectivamente, essa patologia é mundialmente a mais comum entre as mulheres, excluindo-se o câncer de pele não melanoma. São sinais sugestivos: nódulo mamário endurecido, geralmente indolor e fixo, retração da pele em "casca de laranja", perda de liquido ou alteração mamilar, nódulos em pescoço ou axilas (2).

O Swedish Two-County Trial, em 2011, primeiro estudo a demonstrar redução na mortalidade por câncer de mama apenas pelo rastreio mamográfico, exibiu diminuição em 31\% em 29 anos de seguimento no espectro dos 40-74 anos (3). Outrossim, um painel independente de revisão britânico, em 2012, após meta-análise de 11 ensaios clínicos randomizados, concluiu diminuição do risco relativo de mortalidade por câncer de mama de 20\% dentre mulheres que realizaram mamografia majoritariamente entre os 50 e 69 anos (4). Ademais, revisão realizada pela Cochrane em 2013, avaliando 11 estudos com n=616.327, identificou subtração de 19\% na mortalidade por câncer de mama (5).

Visto a importância do rastreamento para o câncer de mama, o Ministério da Saúde, baseado no Instituto Nacional de Câncer, na sua última diretriz em 2015, recomendou: rastreamento bienal em mulheres entre 50 aos 69 anos; posicionando-se contrário ao rastreamento populacional além desse espectro etário, ponderando que possiveis riscos superariam presumiveis benefícios (6).

Por outro lado, no ano de 2017, o Colégio Brasileiro de Radiologia e Diagnóstico por Imagem, a Sociedade Brasileira de Mastologia e a Federação
Brasileira das Associações de Ginecologia e Obstetricia sugeriram novas recomendações para o rastreamento de mulheres com risco populacional usual para o câncer de mama. Apoiados em estudos internacionais que avaliaram o impacto do rastreamento em mulheres entre 40 e 49 anos especificamente e demonstraram redução na mortalidade por câncer de mama entre 18\% e 38\%. Assim, para as sociedades citadas, as diretrizes nacionais deveriam abranger mulheres entre 40 e 74 anos de idade anualmente (7).

Pelo exposto, se faz imprescindivel conhecermos a população feminina que não procura o rastreio mamográfico e suas características sociodemográficas, uma vez reconhecida a importância de tal política na redução do risco relativo de morte por câncer de mama. O presente trabalho teve por objetivo investigar e comparar a prevalência e os fatores associados à não realização da mamografia - sob a óptica, tanto dos critérios do Ministério da Saúde, quanto das recomendações das Sociedades supracitadas, na cidade do Rio Grande, Rio Grande do Sul, Brasil.

\section{Metodologia}

Estudo de delineamento transversal realizado a partir do consórcio de pesquisa "Saúde da População Rio Grandina", composto por estudantes de Pós-graduação de uma Universidade Federal no Sul do Brasil. Localizado no extremo Sul do estado do Rio Grande do Sul, o municipio possui aproximadamente 200 mil habitantes, destes, cerca de 95\% residentes da zona urbana, economicamente concentra-se na atividade portuária, possui Índice de Desenvolvimento Humano de 0,744 pelo censo de 2010 do Instituto Brasileiro de Geografia e Estatística e Produto Interno Bruto per capita de $36.816,67$, segundo análise do Instituto Brasileiro de Geografia e Estatística em 2016 (8). Ademais, dispõe de dois hospitais - um totalmente voltado ao Sistema Único de Saúde, e 32 Unidades Básicas de Saúde (9).

A população estudada compreendeu indivíduos com 18 anos ou mais residentes da zona urbana do município, excluidos institucionalizados em asilos, hospitais, presídios ou com incapacida- 
de física/cognitiva impeditiva para responder ao questionário. No presente trabalho, restringiram-se as análises para individuos do sexo feminino entre 40 e 74 anos de idade.

A amostragem foi constituída em dois estágios. Primeiro considerou-se os setores censitários e, após, os domicílios e os individuos. Baseado no número de 1420 individuos, considerando média de duas pessoas com pelos menos 18 anos por residência - chegou-se a 710 habitações. A partir disso, deu-se a seleção sistemática, selecionando-se 72 dentre 293 setores censitários elegiveis, correspondendo a $25 \%$ com média de 10 domicílios por setor, preferindo-se selecionar mais setores e menos residências para minimizar o efeito de delineamento. Mais detalhes metodológicos podem ser encontrados em outra publicação (8).

Considerou-se como desfechos: a) nunca ter feito mamografia; e b) cobertura de mamografia, conforme dois diferentes critérios. O primeiro, recomendado em conjunto pelo Colégio Brasileiro de Radiologia e Diagnóstico por Imagem, pela Sociedade Brasileira de Mastologia e pela Federação Brasileira das Associações de Ginecologia e Obstetrícia, preconiza rastreamento mamográfico anual para mulheres entre 40 e 74 anos (Categoria A) (7). O segundo, recomendação do Ministério da Saúde que estabelece rastreamento bienal apenas para as mulheres entre 50 e 69 anos (6).

As variáveis independentes incluidas foram: faixa etária, cor de pele, estado civil, escolaridade, nivel econômico, histórico gestacional, tabagismo, obesidade, posse de plano de saúde, consulta ao médico no último ano, cadastro do domicilio na Unidade Básica de Saúde da Familia e se havia recebido visita de agentes de saúde no último ano.

O nivel econômico foi avaliado por meio de análise de componentes principais incluindo posse de alguns bens domésticos e caracteristicas do domicilio, sendo extraido o primeiro componente, que explicou $30 \%$ da variabilidade de todos os itens (eigenvalue $=3,3$ ). Obesidade foi definida como índice de massa corporal (IMC) acima de $30,0 \mathrm{~kg} / \mathrm{m}^{2}$ a partir do peso e altura autorreferidos.

Durante a coleta dos dados, reproduziu-se parcialmente o instrumento em 10,5\% para controle de qualidade dos dados obtendo-se valor médio do índice de kappa das perguntas de 0,80. Todos os questionários foram codificados, revisados e duplamente digitados no programa Epi-Data 3.1 e, os dados transferidos posteriormente para o pacote estatístico Stata 11.2, onde efetuou-se análise exploratória do banco, transformação e categorização das variáveis. A análise univariada utilizou-se de frequências absolutas e relativas. Para análise bivariada utilizou-se teste exato de Fisher e para análise multivariada, regressão de Poisson, considerando-se o efeito de delineamento amostral. Para o ajuste, adotou-se um modelo em três niveis: no primeiro, as variáveis demográficas e socioeconômicas; no segundo, as variáveis comportamentais; e no terceiro, as de serviços de saúde. O nivel de significância estatística considerado foi de $5 \%$ para testes bicaudais. Quando pertinente, reportou-se o valor $\mathrm{p}$ do teste de tendência linear.

O projeto de pesquisa foi aprovado pelo Comitê de Ética em Pesquisa na Área da Saúde da Universidade Federal do Rio Grande, em março de 2016 , parecer 20/2016, sob a resolução 466/12. Todos os participantes do estudo assinaram o Termo de Consentimento Livre e Esclarecido, acordando sua participação. Não alfabetizados consentiram sua participação através da impressão digital no termo após sua leitura em voz alta.

\section{Resultados}

Participaram do estudo 413 mulheres (critério 1) e 246 mulheres (critério 2). Prevalecendo mulheres entre 50 e 59 anos; de cor de pele branca; casadas, separadas ou viúvas; com até oito anos de escolaridade. Cerca de 90\% das mulheres já haviam engravidado, em torno de 20\% eram fumantes correntes e um quarto eram obesas. Aproximadamente metade possuía plano de saúde, $80 \%$ havia consultado um médico no último ano e um terço tinha o domicilio cadastrado em Unidades Básicas de Saúde da Família (Tabela 1). 
TABELA 1 - Descrição de mulheres da zona urbana de Rio Grande, RS, 2016, de acordo com dois critérios (1: exame anual para mulheres de 40 a 74 anos; 2: exame bienal para mulheres de 50 a 69 anos).

Variável

Critério $1(n=413)$

Critério $2(n=246)$

Idade (em anos), $\mathrm{n}(\%)$
$40-49$
$50-59$
$60-69$
$70-74$

Cor da pele branca, $n(\%)$

Estado civil

Casada, viúva, separada ou divorciada

Escolaridade (anos), n(\%)

$$
\begin{aligned}
& \text { o a } 8 \\
& 9 \text { a } 11
\end{aligned}
$$$$
\geq 12
$$

\section{Nivel econômico (tercil), $n(\%)$}

\author{
Mais pobres \\ Intermediárias \\ Mais ricas \\ Já engravidou na vida, n(\%) \\ Fumante atual, n(\%) \\ Obesidade $\left(I M C \geq 30 \mathrm{~kg} / \mathrm{m}^{2}\right)$
}

Plano de saúde, $\mathrm{n}(\%)$

Consultou médico no último ano, n(\%)

Domicilio cadastrado na UBSFr, $n(\%)$

Recebeu visita de agente de saúde, n(\%)

$$
\begin{gathered}
129(31,2) \\
139(33,7) \\
107(25,9) \\
38(9,2)
\end{gathered}
$$$$
359(86,9)
$$

$299(72,4)$

$185(75,2)$

$$
\begin{aligned}
& 209(50,7) \\
& 110(26,7)
\end{aligned}
$$$$
93(22,6)
$$$$
130(52,8)
$$$$
62(25,2)
$$$$
54(22,0)
$$

$\begin{array}{ll}143(34,7) & 92(37,6) \\ 121(29,4) & 63(25,7) \\ 148(35,9) & 90(36,7) \\ 370(89,6) & 223(90,6) \\ 87(21,1) & 59(24,0) \\ 103(26,7) & 57(25,5) \\ 224(54,2) & 138(56,1) \\ 338(81,8) & 200(81,3) \\ 142(34,5) & 70(28,6) \\ 100(24,2) & 54(22,0)\end{array}$

IMC, indice de massa corporal; UBSF, unidade básica de saúde da família.
A prevalência de mulheres que nunca haviam feito mamografia pelo critério 1 foi de $20,3 \%$ (IC95\% $15,9$ a 24,8$)$, oscilando de $11,6 \%$ para as que possuiam plano de saúde a 37,3\% para aquelas que não consultaram um médico no último ano. Conside- rando-se o critério 2, 15,0\% (IC95\% 9,9 a 20,2) das mulheres nunca haviam feito mamografia, variando de $5,3 \%$ entre as obesas a 32,6\% entre aquelas que não consultaram um médico no último ano. 
TABELA 2 - Análise bruta da prevalência (em \%) de mulheres da zona urbana de Rio Grande, Rio Grande do Sul, Brasil, 2016, que nunca fizeram mamografia ou que se incluiam na cobertura de acordo com dois critérios (1: exame anual para mulheres de 40 a 74 anos; 2: exame bienal para mulheres de 50 a 69 anos).

Critério $1(n=413)$

Critério $2(n=246)$

Variável

\begin{tabular}{|c|c|c|c|c|}
\hline & & & & \\
\hline & Nunca fez & Cobertura & Nunca fez & Cobertura \\
\hline Idade & $p=0,01$ & $p=0,28$ & $p=0,37$ & $p=0,59$ \\
\hline $40-49$ & 30,2 & 51,2 & - & - \\
\hline $50-59$ & 13,0 & 51,1 & 13,0 & 66,9 \\
\hline $60-69$ & 17,8 & 50,5 & 17,8 & 63.5 \\
\hline $70-74$ & 21,1 & 34,2 & - & - \\
\hline Cor da pele & $p=0,47$ & $p=0,47$ & $p=0,80$ & $p=0,43$ \\
\hline Branca & 19,8 & 50,1 & 15,5 & 64,3 \\
\hline Preta, parda, amarela & 24,1 & 44,4 & 12,1 & 72,7 \\
\hline Estado civil & $\mathrm{p}<0,01$ & $p=0,05$ & $p=0,30$ & $p=0,44$ \\
\hline Solteira & 33.3 & 41,2 & 19.7 & 66,7 \\
\hline $\begin{array}{l}\text { Casada, viúva, separada } \\
\text { ou divorciada }\end{array}$ & 15,4 & 52,5 & 13.5 & 67,0 \\
\hline Escolaridade (anos) & $p=0,03^{*}$ & $p<0,01^{*}$ & $\mathrm{p}=0,03^{*}$ & $p=0,05^{*}$ \\
\hline o a 8 & 24,4 & 39.7 & 20,0 & 60,0 \\
\hline 9 a 11 & 17,3 & 52,7 & 11,3 & 69,4 \\
\hline$\geq 12$ & 14,0 & 67.7 & 7,4 & 74,1 \\
\hline Nivel econômico (tercil) & $p<0,01^{*}$ & $p<0,01^{*}$ & $p=0,05^{*}$ & $p<0,01^{*}$ \\
\hline Mais pobres & 25.9 & 37,8 & 19,6 & 56,5 \\
\hline Intermediárias & 24,0 & 51,2 & 17,5 & 61,9 \\
\hline Mais ricas & 12,2 & 58,8 & 8,9 & 76,7 \\
\hline Já engravidou na vida & $p=1,00$ & $p=0,63$ & $p=0,36$ & $p=0,65$ \\
\hline Não & 20,9 & 53.5 & 21,7 & 60,9 \\
\hline $\operatorname{Sim}$ & 20,3 & 48,9 & 14,4 & 65.9 \\
\hline Fumante atual & $p=0,01$ & $p=0,07$ & $p=0,10$ & $p=0,09$ \\
\hline Não & 17,5 & 51,8 & 12,8 & 68.5 \\
\hline Sim & 31,0 & 40,2 & 22,0 & 55,9 \\
\hline Obesidade $\left(I M C \geq 30 \mathrm{~kg} / \mathrm{m}^{2}\right)^{\dagger}$ & $p=0,39$ & $p=0,91$ & $p=0,04$ & $p=0,10$ \\
\hline Não & 20,9 & 51,2 & 16,2 & 65.3 \\
\hline Sim & 16,5 & 50,5 & 5.3 & 77,2 \\
\hline Plano de saúde & $p<0,01$ & $p<0,01$ & $p=0,05$ & $p=0,01$ \\
\hline Não & 30,7 & 36,0 & 20,4 & 56,5 \\
\hline Sim & 11,6 & 60,7 & 10,9 & 72,5 \\
\hline
\end{tabular}




\begin{tabular}{lllll}
\hline & \multicolumn{2}{c}{ Critério $\mathbf{1}(\mathbf{n = 4 1 3})$} & Critério 2 ( $\mathbf{n = 2 4 6 )}$ \\
\cline { 2 - 4 } & \multicolumn{1}{c}{ Nunca fez } & Cobertura & Nunca fez & Cobertura \\
\hline Consultou médico no último ano & $p<0,01$ & $p<0,01$ & $p<0,01$ & $p<0,01$ \\
Não & 37,3 & 25,3 & 32,6 & 37,0 \\
Sim & 16,6 & 54,7 & 11,0 & 72,0 \\
Domicilio cadastrado na UBSF & $p=0,44$ & $p=0,30$ & $p=0,69$ & $p=0,55$ \\
Não & 19,3 & 51,5 & 16,0 & 64,0 \\
Sim & 22,5 & 45,8 & 12,9 & 68,6 \\
Recebeu visita de agente de saúde & $p=0,32$ & $p=0,11$ & $p=0,67$ & $p=0,87$ \\
Não & 19,2 & 51,8 & 14,6 & 65,1 \\
Sim & 24,0 & 42,0 & 16,7 & 66,7 \\
Total & 20,3 & 49,4 & 15,0 & 65,5 \\
\hline
\end{tabular}

IMC, indice de massa corporal; UBSF, unidade básica de saúde da família.

* teste de tendência linear.

A cobertura de mamografia pelo critério 1 abrangeu metade das mulheres incluidas, oscilando de um quarto para as que não consultaram um médico no último ano até dois terços para aquelas com 12 anos ou mais de escolaridade. Levando-se em consideração o critério 2, a cobertura alcançou dois terços, indo de um terço entre as que não consultaram um médico no último ano a três quartos entre as obesas (Tabela 2).

Ao analisar os fatores associados, apresentaram maior probabilidade de nunca terem feito mamografia pelo critério 1 os seguintes grupos: mulheres de 40 a 49 anos, solteiras, com até 8 anos de escolaridade, tabagistas, sem plano de saúde e que não haviam consultado um médico no último ano. As mais pobres apresentaram maior chance na análise bruta, mas perdeu associação após ajustes. Por sua vez, os fatores associados à não realização de mamografia pelo critério 2 foram: menor escolaridade, não ter obesidade e não ter consultado um médico no último ano. Menor nível econômico e não ter plano de saúde perderam associação após ajustes (Tabela 2)

Quando se analisou a cobertura de mamografia, os grupos com os maiores índices pelo critério 1 foram: mulheres casadas, separadas ou viúvas, maior escolaridade, detentoras de plano de saúde e que haviam consultado um médico no último ano. As mulheres com maior nivel econômico perderam associação após ajustes. Pelo critério 2, os grupos com maior cobertura foram: mulheres mais ricas e que haviam consultado um médico no último ano. Ter maior escolaridade e possuir plano de saúde esteve associado apenas na análise bruta (Tabela 2).

Destaca-se que a cor da pele, ter engravidado na vida, ter o domicilio cadastrado em Unidade Básica de Saúde da Família e ter recebido visita de agente de saúde não estiveram associados a nenhum dos desfechos (Tabela 2).

\section{Discussão}

Ao investigar e comparar a prevalência e os fatores associados à não realização de mamografia baseado, ou no Ministério da Saúde em 2015, ou nas sociedades de especialistas em 2017, na cidade de Rio Grande, Rio Grande do Sul, observou-se que uma em cada cinco mulheres nunca fizeram o exame pelo critério 1 de cobertura, ao passo que, em torno de uma em cada sete nunca o haviam realizado pelo critério 2 de cobertura. Apresentaram-se como fatores associados a nunca ter feito mamografia: mulhe- 
res mais novas, com menor escolaridade, mais pobres, fumantes, sem plano de saúde e que não haviam ido ao médico no último ano.

Metade da população incluída no critério 1 preencheu os requisitos de cobertura, observando-se que, ser casada, viúva, separada ou divorciada, ter 12 ou mais anos de escolaridade, possuir plano de saúde e ter consultado um médico no último ano, mostraram-se fatores associados a esse desfecho após ajustes. Os fatores relacionados aos dois terços de cobertura entre as mulheres contidas no critério 2 após ajustes foram o tercil econômico mais rico e ter consultado um médico nos últimos 12 meses.

Os programas populacionais devem atingir cobertura mínima de 70\% redução efetiva da mortalidade por câncer de mama (10). Nesse sentido, em 2003, um trabalho, baseado na Pesquisa Mundial de Saúde, apresentou taxa de cobertura no Brasil (analisando a realização do exame nos últimos três anos) de 45.5\% dentre as mulheres com 40 e 49 anos e $48,6 \%$ para a faixa etária dos 50 aos 69 anos (11). Em Singapura, no levantamento após a criação do National Breast Cancer Screening Programme em 2002, a taxa de rastreamento "nos últimos dois anos" passou de cerca de 30\% para em torno de $40 \%$ oito anos depois para a população feminina entre 50 e 69 anos (10). Nos Estado Unidos, a American Cancer Society exibiu em seu relatório anual de 2018 - baseado na National Health Interview Survey de 2005 a 2015, a cobertura referente a mulheres a partir dos 40 anos, estabelecida em torno de 50\% para exames realizados "no último ano", e entre 60 e 70\% para "nos últimos dois anos" (12). Bem como, no consenso europeu de 2017 sobre rastreamento de câncer, as maiores coberturas exibidas para mulheres entre 50 e 69 anos foram na Suécia (76,5\%), na Holanda (77.5\%) e no Reino Unido (83,6\%), todavia, na totalidade, a Europa apresentou 49,2\% - com critérios heterogêneos entre os países (13).

Enquanto na Grã-Bretanha apenas 3\% das 1919 mulheres entre 53-74 anos nunca realizaram mamografia, segundo a National Statistics Omnibus Survey de 2005-2007 (14), nos Estados Unidos, os índices de cobertura são próximos aos exibi- dos em nossos resultados, como apresentado pelo Centers for Disease Control and Prevention, apoiado nos dados da Behavioral Risk Factor Surveillance System de 2012, quando 69\% das mulheres com 45 ou mais haviam realizado mamografia nos últimos dois anos (12), bem como, baseado na National Health Interview Survey, 50 a 55\% das mulheres com pelo menos 40 anos haviam realizado o exame no último ano entre 2005 e 2015, situação próxima ao nosso resultado de $49,4 \%$ de cobertura pelo critério 1 (15).

No contexto brasileiro, ao nosso conhecimento, a maioria dos estudos são ecológicos baseados nos sistemas de informação do SUS e, os índices de comparação disponiveis podem sofrer grande variação. Apoiado na Pesquisa Nacional de Saúde de 2013 estimou-se que 60\% das mulheres entre 50 e 69 anos realizaram mamografia nos últimos dois anos, valor levemente inferior ao nosso achado para o critério 2 (16). Todavia, no mesmo ano, apoiado em dados do Sistema Único de Saúde, dentre as mais de 10 milhões de mamografia realizadas, apenas $25 \%$ foram realizadas na população feminina alvo pelo critério 2, demonstrando verdadeira discrepância nos dados nacionais (17). Estudos baseados na Pesquisa Nacional por Amostra de Domicilio de 2003 apresentaram que 57\% das mulheres com 25 anos ou mais nunca fizeram o exame, ao passo que, ao considerar apenas o espectro entre 50 e 69 anos, esse valor caiu para $49 \%$, sendo o triplo da nossa população $(18,19)$. Em 2007, estudo paulista conduzido com 1302 mulheres com pelo menos 40 anos concluiu que $22 \%$ nunca realizou exame de mamografia, valor próximo ao nosso achado para o critério 1 (20).

Ao estratificarmos as comparações por faixas etárias podemos deferir que entre os grupos dos 40 aos 69 não há diferença significativa na cobertura do rastreio mamográfico, como exibido por estudo baseado na Pesquisa Mundial de Saúde de 2003, apesar da avaliação deles incluir exames dos últimos três anos (11). Dados do Projeto Acesso de 2007 também exibe a faixa dos 50-59 como menor porcentagem de nenhuma realização do exame, entretanto naquela população observou-se que dos 60 anos em diante quase um terço das mulheres 
nunca havia realizado o rastreio, ao passo que nos nossos resultados a mesma proporção é exibida entre os 40-49 anos de idade, diferenças estatisticamente significativas (20). Ao compararmos com o Rio Grande do Sul, dados de 2010 dos sistemas de informação do Sistema Único de Saúde, apresentaram, para as mesmas faixas etárias, desenho de proporções de cobertura semelhante aos por nós apresentados, todavia com menores porcentagens de cobertura a exemplo da faixa acima dos 70 anos a qual se mostrou inferior a metade da nossa população de estudo (21).

Segundo a Pesquisa Mundial de Saúde de 2003, o estado civil da população feminina não foi um fator associado à não realização de mamografia para rastreio de câncer de mama no Brasil. Todavia, assim como exibido em nossos resultados, um estudo paulista demonstrou que dentre aquelas sem parceiro, aproximadamente 1 em cada 3 nunca havia realizado o exame $(11,20)$.

A influência da escolaridade, confirmando nossos resultados, apresenta-se como fator bem estabelecido na literatura vigente $(11,15,16,18,20)$, com verdadeira desigualdade, uma vez que, aquelas com maior escolaridade beiram ou ultrapassam os $70 \%$ de cobertura almejada $(15,16,18)$, enquanto dentre as com menos anos de estudo, a associação com o desfecho pode chegar a cinco vezes mais e, até um terço nunca ter tido acesso ao rastreio (20).

Assim como demonstramos para o critério 2 , outros estudos nacionais confirmam a relação entre maior renda e melhores niveis de cobertura de realização de mamografia nessas mulheres e, também, apontam a influência dessa população possuir trabalho remunerado, inclusive demonstrando que cerca de um quarto das mulheres com 40 anos ou mais que não trabalhavam nunca haviam realizado o exame, valor semelhante ao do nosso trabalho, apesar de termos perdido associação após análise ajustada $(11,18,20)$. A perda de associação do nivel econômico após ajustes revela que a escolaridade pode ter confundido essa associação.

A relação entre o hábito de fumar e a realização de rastreamento mamográfico é escassa na literatura, contudo, análise americana com dados de 2010, 2013 e 2015 da National Health Interview
Survey contendo 15511 mulheres entre 50-74 anos demonstrou que as fumantes possuiam $30 \%$, podendo alcançar em torno de $50 \%$, menos probabilidade de ter realizado o exame em algum momento da vida em relação às mulheres que nunca fumaram, compativel com o que apresentamos, uma vez que na nossa população as fumantes exibiram praticamente probabilidade duas vezes maior de nunca terem realizado mamografia (22). Além disso, no Brasil indivíduos do tercil inferior de escolaridade tem 5 vezes mais chance de serem fumantes quando comparados aos que possuem ensino superior, fato que não só agrega empecilhos socioeconômicos relacionados ao tabagismo como menor acesso à informação, à educação e a assistência à saúde, mas também nesse contexto somam-se fatores que culminam nas menores taxas de cobertura e nos maiores indices dentre as mulheres que nunca fizeram mamografia (23).

Análise baseada na National Health Interview Survey de 1998 com 5277 mulheres entre 50-75 anos demonstrou que entre as caucasianas as de maior IMC tiveram menor probabilidade de realização do rastreio mamográfico no período de dois anos, oposto ao observado dentre as mulheres negras, as quais apresentaram mesma tendência dos nossos resultados, onde as mulheres obesas exibiram maior probabilidade de terem realizado o exame alguma vez na vida no critério 2 (24). Relação que foi considerada como possivelmente necessária, pois mulheres obesas precisam de exames mais frequentes para atingir a mesma taxa de detecção para tumores pequenos do que as de IMC normal (25). Já análise de mais de 100 mil mamografias exibiu que dentre as obesas houve $20 \%$ a mais de risco de falso-positivo, sendo menos específica, embora a sensibilidade não tenha sofrido alterações (26).

Possuir plano de saúde apareceu fortemente associado à realização de mamografia $(11,15,18)$. Esse resultado foi consistente com a Pesquisa Mundial de Saúde de 2003, Surveillance Research de 2013 da American Cancer Society e Inquérito na Baixada Santista de 2007 (11,15,20). Estudo nacional que avaliou condicionantes socioeco- 
nômicos e geográficos relacionados ao acesso à mamografia entre 2003 e 2008 demonstrou que mesmo as maiores taxas de realização sendo entre as mulheres com condições financeiras de arcarem integral/parcialmente ou de estarem vinculada a um plano de saúde, o aumento da abrangência do rastreamento deu-se na camada com renda per capta de até um salário-mínimo, ao passo que o Sistema Único de Saúde financiou 43\% das mamografias no período (19).

A variável mais associada, tanto a nunca ter feito mamografia quanto à cobertura deste exame foi consulta médica no último ano. Na Pesquisa Nacional por Amostra de Domicilios de 2003, a qual incluiu mulheres entre 50-69 anos e considerou intervalo de tempo de 2 anos na realização de mamografia, demonstrou que a partir de uma consulta médica no ano a probabilidade de realização do rastreio aumenta em 30\% a 50\%, podendo atingir duas vezes a chance dessas mulheres o realizarem, uma vez que aumenta a chance de o rastreio ser solicitado. Ressalta-se que a cobertura que apresentamos foi superior (72\%) em relação ao mesmo estudo, que variou de 30 a 55\% conforme o número de consultas no último ano, discrepância que pode ter sido resultado da estratificação realizada no estudo, ao passo que nós analisamos ter ou não consultado apenas (18). Ademais, a racional de que ao buscar a consulta médica mais frequentemente, essa paciente ou demanda por cuidados preventivos com sua saúde ou por conta de alguma adversidade, oportuniza-se a ela a inclusão da mamografia às investigações e às condutas dispensadas.

Como limitação deste estudo, menciona-se o fato de o relato de mamografia ter sido autorreferido, portanto, sujeito a viés de recordatório. Por não ter sido um estudo apenas com esse enfoque não foi possivel aprofundamento em relação ao exame de mamografia no questionário, como a justificativa da requisição e dos resultados, por exemplo. Somado a isso, sugerimos nas próximas investigações interrogar outros fatores como o uso de anticoncepcional oral e, às mulheres que possuem filhos, se amamentaram ou não.

Como pontos fortes, salientamos que este é o primeiro estudo, ao nosso conhecimento, a comparar a cobertura de mamografia usando diferentes critérios vigentes no país. E que, conforme o critério empregado, muda não só a cobertura, mas também os fatores associados.

\section{Conclusão}

Após investigarmos a prevalência e os fatores associados à realização de mamografia, dentro dos dois critérios supracitados, observamos que metade das mulheres preencheram os requisitos pelo critério 1, estando associados, após controle de fatores de confusão, a ter ou já ter tido um cônjuge, ter 12 anos ou mais de estudo formal, estar vinculada a um plano de saúde e ter consultado um médico nos 12 meses antecedentes à entrevista. Ao passo que, dois terços incluíram-se no critério 2 de cobertura, cuja associação mostrou-se estatisticamente significativa quanto ao grupo de tercil econômico mais rico e a ter consultado um médico nos últimos 12 meses após ajustes.

\section{Notas}

\section{Apoio financeiro}

Esta pesquisa foi financiada pela Fundação de Amparo à Pesquisa do Estado do Rio Grande do Sul (FAPERGS) - Programa de Iniciação Científica - ARD / PPP 2014, sob concessão 16/2551-0000359-9).

\section{Declaração de conflito de interesses}

Os autores declaram não haver conflitos de interesses relevantes ao conteúdo deste estudo.

\section{Contribuições dos autores}

Todos os autores fizeram contribuições substanciais para concepção, ou delineamento, ou aquisição, ou análise ou interpretação de dados; e redação do trabalho ou revisão crítica; e aprovação final da versão para publicação.

\section{Disponibilidade dos dados e responsabilidade pelos resultados}

Todos os autores declaram ter tido total acesso aos dados obtidos e assumem completa responsabilidade pela integridade destes resultados. 


\section{Agradecimentos}

Os autores agradecem à Fundação de Amparo à Pesquisa do Estado do Rio Grande do Sul (FAPERGS) por financiar a bolsa de iniciação científica de um dos autores (KFDM) e ao Conselho Nacional de Desenvolvimento Científico e Tecnológico (CNPq) pela bolsa de produtividade em pesquisa do autor SCD.

\section{Referências}

1. World Health Organization. Early diagnosis and screening: Breast Cancer [Online]. 2019 lacesso em 10 mai 2019]. Disponivel em: http://www.who.int/cancer/ prevention/diagnosis-screening/breast-cancer/en/

2. Brasil. A mulher e o câncer de mama no Brasil. Instituto Nacional de Câncer José Alencar Gomes da Silva, Coordenação Geral de Prevenção e Vigilância, Divisão de Detecção Precoce e Apoio à Organização de Rede - 3. ed. rev. atual. Rio de Janeiro: INCA. 2018 lacesso em 7 mai 2019]. Disponivel em: https://www.inca.gov. br/sites/ufu.sti.inca.local/files//media/document// catalogo-expo-mama-3a-ed-2018.pdf

3. Tabár L, Vitak B, Chen TH, Yen AM, Cohen A, Tot T, Chiu SY, Chen SL, Fann JC, Rosell J, Fohlin H, Smith RA, Duffy SW. Swedish Two-County Trial: Impact of Mammographic Screening on Breast Cancer Mortality during 3 decades. Radiology 2011; 260:3. https://doi. org/10.1148/radiol.11110469

4. Independent UK Panel on Breast Cancer Screening. The benefits and harms of breast cancer screening: an independent review. Lancet. 2012; 380: 1778-86. http:// dx.doi.org/10.1016/S0140-6736(12)61611-0

5. Gøtzsche PC, Jørgensen KJ. Screening for breast cancer with mammography. Cochrane Database of Systematic Reviews. 2013; 6. CD001877. https://doi. org/10.1002/14651858.CD001877.pub5

6. Brasil. Diretrizes para a detecção precoce do câncer de mama no Brasil. Instituto Nacional de Câncer José Alencar Gomes da Silva - Rio de Janeiro: INCA. 2015 [acesso em 12 jun 2019]. Disponivel em: https://WwW. inca.gov.br/controle-do-cancer-de-mama/acoes-de-controle/deteccao-precoce

7. Urban LABD, Chala LF, Bauab SP, Schaefer MB, Santos RP, Maranhã NMA, Kefalas AL, Kalaf JM, Ferreira CAP, Canella EO, Peixoto JE, Amorim HLE, Junior HBAC. Recomendações do Colégio Brasileiro de Radiologia e Diagnóstico por Imagem, da Sociedade Brasileira de Mastologia e da Federação Brasileira das Associações de Ginecologia e Obstetrícia para o rastreamento do câncer de mama. Radio Bras. 2017; 50(4): 244-49. http:// dx.doi.org/10.1590/0100-3984.2017-0069
8. Dumith SC, Paulitsch RG, Carpena MX, Muraro MFR, Simões MO, Machado KP, Dias MS, Kretschmer AC, Oliz MM, Pontes LS, Susin LRO. Planejamento e execução de um inquérito populacional de saúde por meio de consórcio de pesquisa multidisciplinar. Sci Med. 2018; 28(3): ID30407. http://dx.doi.org/10.15448/1980-6108.2018.3.30407

9. Oliz MM, Dumith SC, Knuth AG. Utilização de serviços de educação física por adultos e idosos no extremo sul do Brasil: Estudo de base populacional. Ciênc. Saúde Coletiva. 2020; 25(2): 541-52 https://doi. org/10.1590/1413-81232020252.14692018.

10. Loy EY, Molinar D, Chow KY, Fock C. National Breast Cancer Screening Programme, Singapure: Evaluation of participation and performance indicators. J Med. Screen. 2015; 22(4): 194-00. https://doi. org/10.1177/0969141315589644

11. Szwarcwald CL, Leal MC, Gouveia GC, Souza WV Desigualdades socioeconômicas em saúde no Brasil: resultados da Pesquisa Mundial de Saúde, 2003. Rev. Bras. Saúde Matern. Infant. 2005: 5(1): S11-S22.

12. Smith RA, Andrews KS, Brooks D, Fedewa SA, Manassaram-Baptiste D, Saslow D, Brawley OW, Wender RC. Cancer Screening in the United States, 2018: A Review of Current American Cancer Society Guidelines and Current Issues in Cancer Screening. Ca Cancer J Clin. 2018; 68: 297-16. https://doi.org/10.3322/caac.21446

13. The European Union. Cancer Screening in the European Union (2017): Report on the implementation of the Council Recommendation on cancer screening. 2017 [acesso em 10 mai 2019]. Disponivel em: https:// ec.europa.eu/health/sites/health/files/major_chronic_diseases/docs/2017_cancerscreening_2ndreportimplementation_en.pdf

14. Moser K, Patnick J, Beral V. Inequalities in reported use of breast and cervical screening in Great Britain: analysis of cross sectional survey data. BMJ. 2009; 338. https://doi.org/10.1136/bmj.b2025

15. American Cancer Society. Breast Cancer Facts \& Figures 2015-2016. Atlanta: American Cancer Society, Inc. 2015.

16. Freitas JA, Oliveira BGP, Ferreira HH, Espirito Santo $\mathrm{SR}$, Santos RH. Análise do índice de cobertura da mamografia em mulheres entre 50 e 69 anos, por nivel de ensino, segundo unidade de federação. Rev. Med UFC. 2016; 56(1): 14-7. https://doi.org/10.20513/ 2447-6595.2016v56n1p14-17

17. Freitas-Junior R, Rodrigues DCN, Corrêa RS, Peixoto JE, Oliveira HVCG, Rahal RMS. Contribuição do Sistema Único de Saúde no rastreamento mamográfico no Brasil, 2013. Radio Bras. 2016; 49(5): 305-10. http:// dx.doi.org/10.1590/0100-3984.2014.0129

18. Lima-costa MF, Matos DL. Prevalência e fatores associados à realização da mamografia na faixa etária de 50-69 anos: um estudo baseado na Pesquisa Nacional por Amostra de Domicilios (2003). Cad. Saúde Pública. 2007; 23(7): 1665-73. https://doi.org/10.1590/ S0102-311X2007000700018 
19. Oliveira EXG, Pinheiro RS, Melo ECP, Carvalho MS. Condicionantes socioeconômicos e geográficos do acesso à mamografia no Brasil, 2003-2008. Ciência \& Saúde Coletiva. 2011; 16(9): 3649-64. https://doi. org/10.1590/S1413-81232011001000002

20. São Paulo. Instituto de Saúde. Acesso aos serviços de saúde em Municipios da Baixada Santista [Online]. 2008 [acesso 18 jul. 2019]. Disponivel em: http://www. saude.sp.gov.br/resources/instituto-de-saude/homepage/temas-saude-coletiva/pdfs/temas08.pdf

21. Silva GA, Bustamante-Teixeira MT, Aquino EML, Tomazelli JG, dos-Santos-Silva I. Acesso à detecção precoce do câncer de mama no Sistema Único de Saúde: uma análise a partir dos dados do Sistema de Informações em Saúde. Cad. Saúde Püblica. 2014; 30(7): 1537-50. http://dx.doi.org/10.1590/0102-311X00156513

22. Sanford NN, Sher DJ, Butler S, XuX, Ahn C, D'Amico AV, Rebbeck T, Aizer AA, Mahal BA. Cancer Screening Patterns Among Current, Former, and Never Smokers in the Unite States, 2010-2015. JAMA Network Open. 2019; 2(5): e193759. https://doi.org/10.1001/jamanetworkopen.2019.3759

23. Borges MTT, Barbosa RHS. As marcas de gênero no fumar feminino: uma aproximação sociológica do tabagismo em mulheres. Ciência \& Saúde Coletiva. 2009; 14(4): 112939. https://doi.org/10.1590/S1413-81232009000400019.

24. Wee CC, McCarthy EP, Davis RB, Phillips RS. Obesity and Breast Cancer Screening: The influence of Race, Illness Burden, and Other Factors. J Gen Intern Med. 2004; 19: 324-31. https://doi.org/10.1111/j.1525-1497.2004.30354.x

25. Shafaee MN, Gerberding A. Rising Obesity and Breast Cancer Screening. JOJ Pub Health. 2018; 3(4): JOJPH.MS.ID.555616. https://dx.doi.org/10.19080/JO$\mathrm{JPH} .2018 .03 .555616$

26. Elmore JG, Carney PA, Abraham LA, Barlow WE, Egger JR, Fosse JS, Cutter GR, Hendrick RE, D'Orsi CJ, Paliwal P, Taplin SH. The Association Between Obesity and Screening Mammography Accuracy. Arch intern med. 2004: 164(10): 1140-7 https://doi.org/10.1001/ archinte.164.10.1140

\section{Kevin Francisco Durigon Meneghini}

Estudante de Medicina pela Universidade Federal do Rio Grande (FURG), em Rio Grande, RS, Brasil.

\section{Arnildo Agostinho Hackenhaar}

Doutor em Saúde e Comportamento pela Universidade Católica de Pelotas (UCPel), em Pelotas, RS, Brasil; professor da Universidade Federal do Rio Grande (FURG), em Rio Grande, RS, Brasil.

\section{Samuel Carvalho Dumith}

Pós-doutorado em Epidemiologia pela Universidade Federal de Pelotas (UFPel), Pelotas, RS, Brasil; professor da Universidade Federal do Rio Grande (FURG), Rio Grande, RS, Brasil.

\section{Endereço para correspondência}

Samuel Carvalho Dumith

Faculdade de Medicina da Universidade Federal do Rio Grande

Rua Visconde de Paranaguá, 102

Centro, 96203900

Rio Grande, RS, Brasil 